\title{
Covid-19, Coronavirus and Mental Health Rehabilitation at Times of Crisis
}

\author{
Santosh K. Chaturvedi
}

Published online: 2 April 2020

(C) Springer Nature India Private Limited 2020

The ongoing concerns related to the spread of Covid19 infections have enforced a number of ways by which the spread of the virus is curtailed or prevented. These include social distancing, washing hands frequently with soap and water or cleaning these with a hand sanitizer, use of face masks by those who have symptoms or are at risk, and avoidance of touching face, nose, eyes with hands. Social distancing also includes quarantine [home or hospital] and selfquarantine and avoiding large gatherings, working from home and similar methods.

The obvious mental health problems which have become evident due to these measures are the public scare, fears and anxieties. These manifest as health anxieties over touching any potentially infected surface, meeting a person with a travel history involving visit to highly infected places, or developing any signs or symptoms of upper respiratory tract infections [coughing, sneezing, fever]. There is paranoia and doubt if any other person sneezes or coughs in the person's vicinity. While these mental health fears and anxieties are likely to be mild and short lasting, the impact on persons undergoing rehabilitation are likely to be significant.

S. K. Chaturvedi ( $\square)$

Department of Psychiatry, Psychiatric Rehabilitation

Services, National Institute of Mental Health and

Neurosciences, Bangalore, India

e-mail: skchatur@gmail.com
As a result of the advisory on social distancing, day boarding and day care centres for persons with mental health problems have been closed down, for the time being. The health care facilities have been advised to provide only emergency medical care, and people with other health problems, including mental health problems, can wait till the prevailing Covid-19 related situation improves. Hence, persons with chronic mental health problems who need regular medical care are deprived, as they do not have an emergency situation. Hence, mental health rehabilitation being a non-emergency service has also been stopped affecting persons with chronic mental illnesses and intellectual disabilities to be deprived of these services. The work from home conundrum may be useful for persons working in IT, ITes, other industrial sectors but for persons undergoing psychiatric rehabilitation it is quite an antithesis. The vocational training efforts are likely to be affected, and retraining may become a challenge. However, this can be minimised to a certain extent by home based rehabilitation strategies, provided these are planned well in advance.

The philosophy of mental health rehabilitation focuses on helping the person deal with their social skills deficits through social skills training and encouraging social interactions and reducing social distances. The home quarantine and closing down of day care facilities is likely to have a negative impact on their clinical outcome with a risk of aggravation of symptoms and even a relapse. Having to stay at home 
will not only slow down the progress in social skills developments, but reduce their self reliance and self confidence, and affect their vocational potentials. One of the indications of day care is to reduce the negative impact of expressed emotions from family members by minimising face to face contact between person with a mental health problem and their family members. Being home bound, the expressed emotions are likely to worsen again enhancing the risk of aggravation of mental health issues and risk of relapse. The routine of activities of daily living established through the rehabilitation methods will be disrupted, which may take a long time to be restored. The additional, unexpected burden on family caregivers would enhance their caregiver stress and affect the care giving.

There are limited activities which persons with or without mental health problems can engage in at home. This is likely to enhance their use of mobile phones, internet and use of social media to keep themselves engaged. Whether this would increase behavioural and internet addictions, only the coming days will tell.

Other psychiatric disorders which are likely to be adversely affected are health and illness anxiety disorders, social anxiety and phobic disorders, generalized anxiety disorders, somatoform disorders and abnormal illness behaviours, obsessive compulsive disorders [due to repeated hand washing], alcoholism and substance use disorders, eating disorders and other life style related disorders. Similar challenges are likely to be faced by children and adolescents, and elderly with mental health problems and disorders. There may be some truth in these apprehensions and experiences, as these measures of social distancing and quarantine have changed life styles significantly.

It is also a challenge to make mental health professionals; specially psychiatrists, keep their hands off their face, chin and mouth, and break their lifelong habits and mannerisms! Social media is abuzz with jokes, wisecracks and light hearted humour on people being confined to home space indefinitely and getting on each other's nerves.
Thus, it is quite a challenging situation. On one hand, it is the safety and precautions against the Covid19 virus for persons receiving rehabilitation and other general public, on the other hand the impact of these precautionary methods on the mental health recovery, care and rehabilitation of persons receiving mental health interventions. Health care systems were not prepared for this outbreak, even less so about its impact on mental health and persons with mental illnesses, and those in the process of rehabilitation. Suitable rehabilitation measures should continue without compromising caution and physical risk of infection. It is both an ethical dilemma and a public health challenge. Professionals should be prepared in advance for any potential crises like situations, like this one. Such preparedness would ensure continual rehabilitation without compromising safety and medical risks.

Under the cloud of this pandemic, the eleventh issue of the journal is unique in many ways. From this year, there will be three issues in a year. This issue has manuscripts from different parts of the world-Ghana, Japan, Australia, United Kingdom, USA, Switzerland and India. One of these draws attentions to the refreshing use of therapeutic humour and comedy movie watching. There are a couple of articles on persons with autistic spectrum disorders, one on use of social skills training and another on empowerment programme for parents of persons with high functioning autistic spectrum disorders from Japan. Other informative articles are on cognitive remediation in schizophrenia in rural Australia, non-Cartesian view on suicide and suicide prevention from Switzerland, post natal depression from Ghana, risk assessments and third sector mental health recovery programmes from UK.

Hopefully, by the time the next issue of the journal is released, the clouds of Covid-19 would have blown away. Till then, stay safe.

Publisher's Note Springer Nature remains neutral with regard to jurisdictional claims in published maps and institutional affiliations. 